mass screening is much less practicable-on average only $0.1 \%$ of such asymptomatic individuals will have early gastric cancer.

Total gastrectomy is considered to be the treatment of choice by most, but not all, authors. It is clear that many problems remain. but this book succeeds admirably in its primary aim of raising the level of awareness in the U.K. for early gastric cancer and it should be read by everyone with an interest in gastroenterology.

T. W. WARNES.

University Department of Gastroenterology. Manchester Royal Infirmary. Manchester M8 7PL.

\section{Epidemiology of Diseases}

Edited by D. L. Mil.ter and R. D. T. FARMer. Pp. xiii +492. illustrated. Blackwell Scientific Publications, Oxford, 1982. $£ 28.50$.

While there are by now several textbooks on the methods and general applications of epidemiology, few. if any, have so far attempted systematically to describe the epidemiology of the major diseases. Anything of this kind would be an ambitious undertaking and is what the editors of this volume set out to do. How far have they succeeded? The book is intended not only for those in preventive medicine but for practising clinicians as well. The 9 main sections cover infectious diseases, diseases of the heart and blood. chronic respiratory disease, digestive disease. diseases of the genitourinary system and female breast. endocrine diseases. locomotor and neurological diseases. accidents and suicide and maternal and fetal health. The list of authors includes both epidemiologists and clinicians: on the whole. the former provide the most useful contributions. Dr Jocelyn Chamberlain's chapter on cancer of the female breast, for example. provides an account not only of the general epidemiology of the condition and of aetiological hypotheses, but also of progress in attempts at prevention through screening. The chapter on rheumatic disorders by Dr Philip Wood and Dr Elizabeth Badley is a valuable and largely self-contained account of a particularly difficult topic. Some of the chapters in the sections on gastro-intestinal and genito-urinary disease are on the sketchy side. However, the sections as a whole are well-referenced. many including suggestions for further reading. Consequently, the book is a useful starting-point for anyone unfamiliar with the epidemiology of a particular condition. and. in some cases, it provides a definitive account.

T. W. ME.ADE.

MRC Epidemiology and Medical Care Unit. Northwick Park Hospital.

Harrow. Middlesex HAI 3 UJ.

\section{Evaluation of Methods of Treatment and Diagnostic Procedures in Cancer. Studies conducted by the Project on Controlled Therapeutic Trials (1978-1982)}

Edited by R. FLAMANT and C. FOHANNO. UICC Technical Report Series-Vol. 70. Pp. 146. UICC. Geneva. Hans Huber, Bern. 1982. S.Fr. 24.00.

This excellent short paperback. printed cheaply using typewriter script, is a collection of nine papers prepared for the UICC. Each article addresses a problem in the assessment of methods of cancer treatment. There is discussion of the ethics and design of controlled trials, the evaluation of new diagnostic tests, of the statistical consideration in trial design and problems in informed consent.

The articles are a model of lucidity. common sense and practicality. Any oncologist involved in trials of treatment should read and understand what is in this little book. It only takes $2 \mathrm{hr}$ to read and it is well worth putting this time aside to remind oneself of the problems of this type of scientific investigation. Highly recommended.

R. L. Souhami.

Faculty of Clinical Sciences.

University College.

London WCI.

\section{Guidelines for a Voluntary Cancer Organization}

Edited by M. A. WooD. UICC Technical Report Series. Vol. 65 Pp. 162. UICC. Geneva, Hans Huber. Bern, 1982. Sw.Fr. 28.00.

With the increasing interest and concern in matters of health. a number of voluntary organisations have been set up which are involved with specific diseases. These organisations are usually run by lay people, though generally in collaboration with interested members of the medical profession.

Provided they are sensibly administered, these societies can do nothing but good. From the point of view of the patient. they can offer a wide range of help. they can create a link between the doctor and the public so that each side knows each other's problems. they can play a very important part in fund raising and finally they provide a most useful outlet for those people who wish to help those in trouble.

This booklet. published by the UICC. is a most useful guide for setting up such voluntary organisations. Medical practice and the social scene vary in different countries and. although this book is written by an international team who have wide experience in this type of work. some of the suggestions and advice will not be universally applicable. It deals in a clear and practical way with the problems which can arise in initiating a voluntary organisation. It starts with an important chapter on organisational structure. it goes on to discuss relationships to health care. research. welfare. education, public relations and finally fund-raising. It is full of helpful advice. useful check lists etc and it cannot be too highly recommended to those who are considering working in this field.

J. R. TROUNCE.

Guy`s Hospital.

London SEI 9LT.

\section{An Introduction to Neurophysiology}

By J. F. StEIN. Pp. viii +386 , illustrated. Blackwell Scientific Publications, Oxford. 1982. $£ 12.00$.

This relatively small volume sets out to present neurophysiology in a lively and exciting way. The author has not always succeeded in conveying the fascination of the subject. as he states is his intention. but it is always very readable. There is inevitably, a degree of simplification in a volume of this small size and it is useful to have some well chosen further readings given at the end. These are mainly of other books: perhaps a few more original papers should be included.

The layout is relatively standard. conveying the properties of neurones. synaptic transmission. sensory mechanisms. special senses. motor control. spinal reflexes and the extrapyramidal system. These chapters. the main body of the book. present an adequate account but are followed by several short chapters on sleep. specialised cortical function and mechanisms of memory. which barely scratch the surface of the vast amount of data now available. incomplete or cuntradictory as some of it may be. It is easy to understand why these chapters were so rigorously pruned from the author's statement of intention in the preface but it has been over-done. This section inevitably contains some controversial facts and a few errors creep in. such as REM sleep scalp activity being shown as similar to Stage $I$ sleep and an auditory-evoked potential without polarity indication. Others in my own field of clinical neurophysiology will find the very brief and inadequate paragraph on clinical uses of EEG saddening. Here a fellow scientist seems to confuse the physiological information of the EEG with the anatomical precision of CT scanning. while ignoring the many aspects of clinical disease in which the EEG may be dramatically altered without anatomical change. I am sure Dr Stein does not really confuse these issues but it illustrates how the later chapters have suffered by being over-brief. Better omitted altogether than sacrificing academic standards to this extent. The inclusion of 2 chapters at the beginning on neuroanatomical and neurophysiological techniques is a welcome innovation and one particularly appreciated by students who have seen the book.

Overall. the impression is of a book containing much of value in very readable style and which would make a good introductory text. 
but medical students and undergraduates would need to progress to a larger, more complete work. It would be of value as a general overview of neurophysiology for people working in related fields such as medical physicists, laboratory technicians, speech therapists and similar professions.

S. G. BAYLISS,

Royal Surrey County Hospital, Guildford, Surrey.

\section{Leukaemia}

Edited by N. L. WARner and D. METCALF. A series of workshops on the Biology of Human Cancer. Report No. 14. UICC Technical Reports Series Vol. 61. Pp. 232. International Union Against Cancer, Geneva, 1982. (Distributed by Hans Huber, Bern, Switzerland). Sw.Fr. 44.00 .

Leukaemia must surely be one of the most exciting areas in which a medical scientist could currently be involved. The dramatic advances in treatment over the last 2 or 3 decades are now being mirrored by an understanding of the basic cellular and sub-cellular mechanisms. Indeed, new bone marrows are being exchanged for old! So how can this subject be turned into such heavy reading? The answer can only be, by committee.

Fortunately, the content of this particular Workshop is excellent, with a comprehensive review of the biology of both leukaemic cells in man and sub-human species and their normal equivalents, by some of the most outstanding workers in the field. Obviously, there must be advantages in publishing the proceedings of Workshops. This is clearly seen in the way in which both sides of the argument are presented, this being best illustrated in the section on aetiology of leukaemia. In fact, it is this last section in which some of the most recent and potentially useful developments have been made, especially the identification by Gallo and his co-workers, at long last, of a virus which appears to have a definite role in the development of an albeit rare type of human leukaemia. This work is extremely topical and of great interest, especially the probable role of virus in human oncogenic expression and the relationship of products of those oncogenes to cell proliferation.

Many other topics are dealt with, including the application of immunology to the diagnosis of leukaemia and a comprehensive review of cytogenetics in leukaemia, a topic likely to become more important in the future for identification of patients likely to perform poorly with conventional therapy. A further section dealing with the recognised patterns of leukaemia cell growth in vitro speculates about the possible future benefit of predictive tests of leukaemia sensitivity to various therapeutic agents based on the in vitro sensitivity of cultured leukaemic cells. On this topic, the clinician is yet to be convinced.

For me, the most exciting section was certainly that on the aetiology of leukaemia with the often controversial evidence for the role of such diverse agents as radiation and virus being comprehensibly reviewed.

This publication should certainly be of interest to both basic scientists and clinicians working in the field of leukaemia, one of the major attractions being the extensive bibliography with 581 references.

\section{H. Grant Prentice, Royal Free Hospital, London NW3 2QG.}

\section{Manson's Tropical Diseases}

By P. E. C. Manson-BAhr and F. I. C. APTED. 18th edn. Pp. 843, illustrated. Bailliere Tindall, London, 1982. $£ 30.00$.

Is tropical medicine synonymous with medicine in the tropics? Most people who have worked in the tropics would deny this, and would restrict the use of the term 'tropical diseases' to those diseases which, by virtue of biological, climatic, social or environmental conditions occur exclusively or predominantly in the tropics and subtropics. Manson's original work, first published in 1898, was deliberately named a manual, 'of handy size; for the exigencies of travel and of tropical life are, as a rule, incompatible with big volumes'. Clearly, the situation has changed and this, the 18 th edition, continues pattern of growth of standard textbooks. The 18 th edition weighs $\mathrm{kg}$, compared to $1.75 \mathrm{~kg}$ for the 17 th edition. The contents are arranged in the same basic style as in previous editions; a shot introduction on non-infective disorders and anaemia in the tropicsos followed by sections of aetiologically organized descriptions 9 tropical diseases; protozoal, helminthic, viral, bacterial, spirochaetai rickettsial, fungal. The tropical venereal diseases lymphogranulorma venereum and granuloma inguinale are included here. The last $\overrightarrow{5}$ sections deal with diseases of the environment, nutritional disorde venous and poisons, ophthalmology, and drugs. There are appendices, 186 pages long, dealing with detailed descriptif protozoology, helminthology and entomology. The book purports serve two aims; as a textbook for students and as a general referengef book for practitioners in the field.

Students, I believe, will find too much here: a smaller manuag dealing with clinical parasitology would be all that is required (even for students in tropical countries). If only the truly tropical diseases had been included, the book would have been first class. Howevef the introduction of non-tropical (but none the less important) topis has led to serious imbalances and omissions. Pulmonary tuberculo sis, perhaps the most important single tropical disease after malarin. is dealt with in 4 pages, while brucellosis, an unimportant disease in the tropics, receives 7 pages. Gonorrhoea, of huge importance, is nof mentioned, hepatitis is summarily dealt with in $2 \frac{1}{2}$ pages (and excludes virtually all the clinical aspects of hepatitis B). Poliomyelifis is dismissed in one page, and measles in 2 and so on. The section 迥 drugs includes some curious compounds (Glaucarubin, pelletierine. stilbazium, etc), which even the author admits are not generaliy available, so why include them?

The authors are clearly confused: is this to be a textbook of tropical diseases, or a textbook of medicine in the tropics? If the former, it is too extensive, if the latter, too imbalanced.

Now, the good news. The publishers have radically altered the format of the book. The cover contains a striking photomicrograre of the head of a mosquito: the book is larger in size, the prett arranged in 2 columns and much easier to scan, the quality of the line drawings is excellent: numerous, new black and white p graphs have been included: the references have been updated. cost $(£ 30)$ seems reasonable for a book of this size.

Clearly, this is a textbook which should be on the shelves of an medical library. If (when) I return to the tropics, I might pack it my sea-mail, but it will not be a priority book for my travellip library.

G. H. REE, Hospital for Tropical Diseases, London NWI OPE

\section{Medical Data Interpretation For MRCP}

By Roger Gabriel and Cynthia M. Gabriel. 2nd edn. Pp. xī 210, illustrated. Butterworths, London, Boston, Sydney, Wellington, Durban, Toronto, 1982. £3.95.

This second edition maintains the high standards which made the first so popular and helpful for MRCP candidates. There are chapters of questions covering all systems and the final chapter provides the answers with helpful explanations attached to these answers.

If a candidate masters the 300 interpretations in this book, should have no difficulty in the Data section of the MRC. examination. It is strongly recommended for just this purpose. $\mathrm{N}$

D. Geraint James, o

Royal Northern Hospital, N London N7. N

\section{The Newborn Child}

By D. G. Vulliamy. 5th edn. Pp. 217, illustrated. Churchidt Livingstone, Edinburgh and London, 1982. £5.95.

This is the 5 th edition of this successful primer of neonatal medicine In the first 4 chapters, the care of the normal newborn is dealt with some detail. There is then a chapter on the preterm infant, including 